Apidologie, 1979, $10(4), 395-401$.

NOTE TECHNIQUE

\title{
APPLICATION AU MIEL D'UNE MÉTHOdE DE DOSAGE PAR VOIE ENZYMATIQUE DES MONOSACCHARIDES RÉDUCTEURS
}

\section{Anwendung einer quantitativen enzymatischen Methode zur Bestimmung reduzierender Zucker auf den Honig}

\author{
M. GONNET
}

Station Expérimentale d'Apiculture - I.N.R.A.

F. 84140 Montfavet

\section{SUMMARY}

\section{A METHOD OF TITRATION OF REDUCING MONOSACCHARIDES BY ENZYMATIC} WAY APPLIED TO HONEY

An enzymic method of titration of glucose and fructose, which can be used for food, was applied to honey. The analyzed samples were honeys of which floral origin was known.

The results were good and they enable us to recommend the use of this method for the research and the routine titration of reducing sugars in honey.

\section{RÉSUMÉ}

Une méthode de dosage enzymatique du glucose et du fructose, utilisable dans les aliments (BoEHRINGER, Mannheim), a été appliquée au miel. Les échantillons analysés sont constitués par des miels d'origine florale connue.

Les résultats obtenus sont satisfaisants et nous permettent de préconiser l'usage de cette méthode pour la recherche et le dosage de routine des sucres réducteurs dans le miel.

\section{INTRODUCTION}

L'analyse quantitative des monosaccharides réducteurs (glucose et fructose) ainsi que la mesure de la teneur en eau sont deux éléments très importants de l'analyse des 
miels. Du rapport glucose/fructose et surtout du rapport glucose/eau vont dépendre en grande partie la rapidité de cristallisation d'un miel ainsi que sa structure cristalline. Avant de procéder aux principales opérations technologiques que sont la pasteurisation, la cristallisation dirigée, le mélange ou la refonte des miels, il faut connaitre ces données essentielles; elles aident au choix du traitement à appliquer.

Les méthodes employées pour le dosage des sucres sont nombreuses; elles font appel à la chimie analytique classique ou à la chromatographie de partage. La méthode chimique officielle utilisée pour le dosage des sucres réducteurs (1977) est la méthode de LUFF-SCHOoRL. Les résultats qu'elle apporte sont globaux : ils ne permettent pas de différencier glucose et fructose. Des tests colorimétriques ont été mis au point (GoNNET, 1973), mais deux dosages distincts sont nécessaires pour obtenir une bonne estimation de chacun des monosaccharides présents dans le miel. Les méthodes de chromatographie quantitative sont aujourd'hui très employées. Elles donnent d'assez bons résultats. Cependant, en règle générale, les manipulations sont longues et délicates. L'analyse par chromatographie en phase gazeuse mise au point par PoURTALLIER (1967) constitue la méthode officielle pour le dosage des différents sucres dans le miel mais la technique est trop délicate pour être adaptée aux conditions de travail du laboratoire d'une grande miellerie. Par ailleurs, elle représente un investissement assez lourd.

Nous avons donc cherché à adapter au miel une méthode de dosage du glucose et $\mathrm{du}$ furctose qui soit rapide, suffisamment précise et utilisable dans un laboratoire ne disposant que d'un équipement léger; ceci pour répondre aux impératifs technologiques cités plus haut. Les dosages par voie enzymatique proposés par BERGMEYER et al. (1970) sont parfaitement adaptés à la solution du problème posé; nous en avons fait l'application au miel.

\section{MATÉRIEL ET MÉTHODES}

Nous reprenons ici les grandes lignes de la fiche technique d'analyse fournie par BoEHRINGER (1978) en y ajoutant cependant quelques précisions que nous jugeons importantes en ce qui concerne l'analyse du miel.

\section{Principe du dosage}

Le glucose et le fructose sont phosphorylés par l'adénosine- $5^{\prime}$ - triphosphate (ATP) en glucose-6phosphate (G-6-P) et fructose-6-phosphate (F-6-P), dans une réaction catalysée (1) (2) par l'hexokinase (HK).

(1) Glucose + ATP $\stackrel{\text { HK }}{\rightleftharpoons}$ G-6-P + ADP (Adénoside-5'-diphosphate)

(2) Fructose + ATP $\stackrel{\text { HK }}{\rightleftharpoons}$ F-6-P + ADP 
En présence de glucose-6-phosphate déhydrogénase (G-6-P-DH), le glucose-6-phosphate est oxydé par le nicotinamide-adénine-dinucléotide-phosphate (NADP) en gluconate-6-phosphate. Il se forme du nicotinamide-adénine-dinucléotide-phosphate réduit (NADPH) (3).

(3) G-6-P + NADP $\underset{\text { G-6-P-DH }}{\rightleftharpoons}$ gluconate-6-phosphate + NADPH + $\mathrm{H}^{+}$

La quantité de NADPH formée au cours de la réaction est proportionnelle à la quantité de glucose. On la détermine par son absorption dans le proche U.V. à $340 \mathrm{~nm}$ ou à $365 \mathrm{~nm}$.

A la fin de la réaction (3), le F-6-P est transformé en G-6-P par la phosphoglucose-isomérase (PGI) (4).

(4) F-6-P PGI G-6-P

Le G-6-P formé réagit à son tour avec le NADP en formant du gluconate-6-phosphate et du NADPH. La quantité de NADPH formée est mesurée à nouveau, elle est proportionnelle à la quantité de fructose. On la détermine de la même manière par son absorption à $340 \mathrm{~nm}$ ou à $365 \mathrm{~nm}$.

\section{Mode opératoire}

Il existe dans le commerce des coffrets (1) prêts à l'utilisation qui contiennent chacun trois flacons permettant d'effectuer vingt-cinq dosages environ :

- le premier de ces flacons (sol. 1) contient un lyophylisat que l'on met en solution dans $27 \mathrm{ml}$ d'eau distillée au moment de l'emploi; c'est un tampon triéthanolamine à $p H$ 7,6 contenant du nicotinamideadénine-dinucléotide-phosphate (NADP) et de l'adénoside triphosphate (ATP);

- le second flacon (sol. 2) contient $0,7 \mathrm{ml}$ de suspension enzymatique composée de : hexokinase (environ $200 \mathrm{U}$ ) et de glucose-6-phosphate-déshydrogénase (env. $100 \mathrm{U}$ );

- le troisième enfin (sol. 3) renferme $0,7 \mathrm{ml}$ de phosphoglucose-isomérase (490 U).

Les prélèvements sont faits à la micropipette ou à la microseringue pour les volumes de 0,1 et $0,02 \mathrm{ml}$. Les réactions sont conduites en cuves de verre de $1 \mathrm{~cm}$ de côté. L'appareil utilisé est un spectrocolorimètre réglé dans le proche UV à $340 \mathrm{~nm}$. Les lectures doivent être effectuées en densité optique. Ces dosages sont faits en série; il est important que dans une même série toutes les cuves de verre soient très propres, homogènes et parfaitement calibrées. Ces cuves, lorsqu'elles contiennent de l'eau distillée doivent toutes permettre de faire le point zéro de l'appareil avec un seul réglage effectué sur la tête de série.

On prépare par dilutions successives une solution contenant $1 \mathrm{mg}$ de miel par millilitre d'eau distillée. Le miel est pesé à partir d'un stock parfaitement homogénéisé. Si l'on observe dans le produit mis en solution quelques impuretés en suspension, la première dilution sera filtrée sur papier après étalonnage. La prise d'essai de $0,1 \mathrm{ml}$ renferme environ $40 \mu \mathrm{g}$ de fructose et $40 \mu \mathrm{g}$ de glucose.

Chaque dosage ou série de dosages est fait contre un témoin dans lequel la solution de miel est remplacée par de l'eau distillée. Le dosage est conduit selon le schéma suivant:

\begin{tabular}{|c|c|c|}
\hline & Témoin & Essai \\
\hline Sol. 1 & $1 \mathrm{ml}$ & $\mathrm{ml}$ \\
\hline Sol. “ Miel ” & - & $0,1 \mathrm{ml}$ \\
\hline Eau distillée & $2 \mathrm{ml}$ & $1,9 \mathrm{ml}$ \\
\hline
\end{tabular}

On mélange et après trois minutes environ on fait la lecture au spectrocolorimètre soit E 1 pour le témoin et $E^{\prime} \mathbf{l}$ pour l'essai. Puis on amorce la réaction en ajoutant dans chaque cuve...
Sol. 2
$0,02 \mathrm{ml}$
$0,02 \mathrm{ml}$

(1) Préparateur : “Boehringer " - Food analysis - Mannheim (Allemagne Fédérale) et Boehringer MannheimFrance S.A., 2, avenue du Vercors, 38240 Meylan. 
On mélange et après dix à quinze minutes on fait la lecture au spectrocolorimètre soit $\mathrm{E} 2$ pour le témoin et $E^{\prime} 2$ pour l'essai. On poursuit la réaction...

$$
\text { Sol. } 3 \quad 0,02 \mathrm{ml} \quad 0,02 \mathrm{ml}
$$

On mélange et après dix à quinze minutes on fait la lecture au spectrocolorimètre soit $\mathrm{E} 3$ pour le témoin et $E^{\prime} 3$ pour l'essai.

La température du laboratoire doit être comprise entre 20 et $25^{\circ} \mathrm{C}$. Si la réaction n'est pas terminée après quinze minutes, on continue la lecture jusqu’à D.O. constante.

Le calcul des résultats s'effectue comme suit :

$\left(E^{\prime} 2-E^{\prime} 1\right)-(E 2-E$ 1) $=\Delta$ E Glucose (exprimé en D.O.)

$\left(E^{\prime} 3-E^{\prime} 2\right)-(E 3-E 2)=\Delta E$ Fructose (exprimé en D.O.)

La formule générale pour le calcul des concentrations est :

$c(\mathrm{~g} / \mathrm{l})=\frac{V \times P M}{\varepsilon \times d \times v \times 1000} \Delta \mathrm{E}$ où :

$V=$ Volume final de l'essai ou du témoin $(\mathrm{ml})$

$v=$ Volume de la prise d'essai miel (ml)

$P M=$ Poids moléculaire de la substance à doser $(180,16$ pour le glucose et le fructose).

$d=$ épaisseur de la cuve

$\varepsilon=$ coefficient d'extinction du NADPH :

$$
\begin{aligned}
& \text { sous } 340 \mathrm{~nm}=6,3\left(1 . \mathrm{m} \mathrm{mole}^{-l} \cdot \mathrm{cm}^{-l}\right) \\
& \text { sous } \mathrm{Hg} 365 \mathrm{~nm}=3,5\left(1 . \mathrm{m} \mathrm{mole}^{-1} \cdot \mathrm{cm}^{-l}\right)
\end{aligned}
$$

soit pour le glucose en grammes par litre de solution de miel : (lecture sous $340 \mathrm{~nm}$ ) :

$$
c=\frac{3,02 \times 180,16}{6,22 \times 1 \times 0,1 \times 1000} \times \Delta \mathrm{E}(\text { glucose })=0,864 \times \Delta \mathrm{E} \text { Glucose }
$$

Soit pour le fructose en grammes par litre de solution de miel : (lecture sous $340 \mathrm{~nm}$ ).

$$
c=\frac{3,04 \times 180,16}{6,22 \times 1 \times 0,1 \times 1000} \times \Delta \mathrm{E}(\text { fructose })=0,869 \times \Delta \text { E Fructose }
$$

Les résultats sont traduits en pour cent de glucose et de fructose dans le miel frais.

\section{RÉSULTATS OBTENUS}

Nous avons effectué des analyses avec répétitions sur une série de onze miels d'origine géographique et florale définies. Les résultats figurent au tableau $\mathrm{n}^{0} 1$.

On note une bonne reproductibilité d'ensemble de ces résultats alors que chaque analyse pour un même miel est pratiquée sur un prélèvement différent effectué à partir d'un lot initial de 250 grammes environ. Si l'on tient compte également des risques d'erreur consécutifs à la forte dilution et au très faible volume de la prise d'essai, l'homogénéité relative des résultats est plus que satisfaisante.

\section{CONCLUSIONS}

La méthode de dosage enzymatique des monosaccharides réducteurs dont nous proposons ici une application au miel est une analyse simple et rapide. Le dosage d'une 
TAB. 1. - Résultats des dosages du glucose et du fructose pratiqués sur 11 échantillons de miel.

TAв. 1. - Resultate der quantitativen Bestimmung von Glukose und Fruktose in 11 Honigproben

\begin{tabular}{|c|c|c|c|c|c|c|c|c|}
\hline \multirow[b]{2}{*}{$\begin{array}{c}\text { Miel } \\
\text { Origine botanique } \\
\text { Honig } \\
\text { Botanische Herkunft }\end{array}$} & \multicolumn{4}{|c|}{$\begin{array}{l}\text { Glucose }(\mathrm{g} / 100 \mathrm{~g} \text { de miel }) \\
\text { Glukose (g/100 g Honig) }\end{array}$} & \multicolumn{4}{|c|}{$\begin{array}{l}\text { Fructose }(\mathrm{g} / 100 \mathrm{~g} \text { de miel }) \\
\text { Fruktose }(\mathrm{g} / 100 \mathrm{~g} \text { Honig }\end{array}$} \\
\hline & $\begin{array}{l}\text { Résultats } \\
\text { Resultate }\end{array}$ & 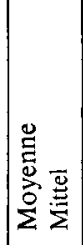 & 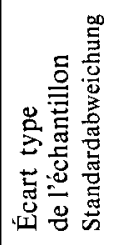 & 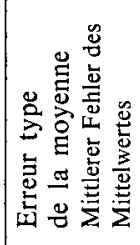 & $\begin{array}{l}\text { Résultats } \\
\text { Resultate }\end{array}$ & 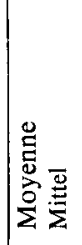 & 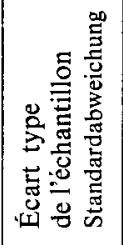 & 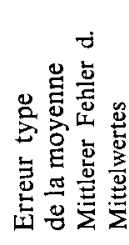 \\
\hline $\begin{array}{l}\text { Robinia pseudacacia } \\
\text { Acacia } \\
\text { Robinie }\end{array}$ & $\begin{array}{ll}27,1 & 25,5 \\
27,3 & 26,1 \\
27,5 & 27,6 \\
27,5 & \end{array}$ & 26,9 & 0,76 & 0,29 & 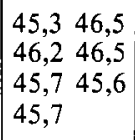 & 45,9 & 0,44 & 0,17 \\
\hline $\begin{array}{l}\text { Calluna vulgaris } \\
\text { Bruyère " callune " } \\
\text { Heidekraut }\end{array}$ & $\begin{array}{ll}32,9 & 31,2 \\
32,1 & 32,0 \\
31,9 & \end{array}$ & 32,0 & 0,54 & 0,24 & $\begin{array}{|ll|}41,5 & 42,6 \\
42,6 & 41,4 \\
41,5 & \end{array}$ & 41,9 & 0,56 & 0,25 \\
\hline $\begin{array}{l}\text { Castanea sativa } \\
\text { Chataîgnier } \\
\text { Edelkastanie }\end{array}$ & $\begin{array}{ll}33,5 & 32,4 \\
33,5 & 32,9 \\
33,0 & \end{array}$ & 33,1 & 0,41 & 0,18 & $\mid \begin{array}{ll}44,0 & 44,4 \\
42,8 & 42,5 \\
43,6\end{array}$ & 43,5 & 0,71 & 0,32 \\
\hline $\begin{array}{l}\text { Brassica napus } \\
\text { Colza } \\
\text { Raps }\end{array}$ & $\begin{array}{|ll|}40,2 & 39,6 \\
42,2 & 40,2 \\
42,6 & 40,1\end{array}$ & 40,8 & 1,14 & 0,47 & $\begin{array}{|ll|}36,9 & 40,2 \\
40,1 & 40,1 \\
38,2 & 39,4\end{array}$ & 39,1 & 1,22 & 0,50 \\
\hline $\begin{array}{l}\text { Lavandula vera } \\
\text { Lavande } \\
\text { Lavendel }\end{array}$ & $\begin{array}{|ll|}33,8 & 33,5 \\
32,8 & 33,2 \\
32,6 & 32,5 \\
32,4 & 33,2\end{array}$ & 33,0 & 0,47 & 0,17 & $\begin{array}{ll}40,1 & 40,5 \\
38,7 & 40,9 \\
39,6 & 39,9 \\
39,0 & 39,4\end{array}$ & 39,8 & 0,69 & 0,24 \\
\hline $\begin{array}{l}\text { Lavandula vera } X \\
\text { Lavandula latifolia } \\
\text { Lavandin } \\
\text { Lavandin }\end{array}$ & $\begin{array}{ll}34,1 & 33,1 \\
33,2 & 32,9 \\
32,3\end{array}$ & 33,1 & 0,58 & 0,26 & $\begin{array}{|ll|}36,2 & 39,7 \\
37,8 & 38,2 \\
37,5 & \end{array}$ & 37,9 & 1,13 & 0,51 \\
\hline $\begin{array}{l}\text { Rosmarinus officinalis } \\
\text { Romarin } \\
\text { Rosmarin }\end{array}$ & \begin{tabular}{ll|}
37,5 & 38,1 \\
37,6 & 36,9 \\
38,1 & 38,5 \\
38,5 &
\end{tabular} & 37,9 & 0,54 & 0,20 & $\begin{array}{ll}39,2 & 41,3 \\
40,4 & 40,1 \\
39,0 & 39,8 \\
39,0 & \end{array}$ & 39,8 & 0,79 & 0,30 \\
\hline $\begin{array}{l}\text { Tilia sp. } \\
\text { Tilleul } \\
\text { Linde }\end{array}$ & $\mid \begin{array}{ll}33,1 & 35,4 \\
35,5 & 34,2 \\
35,4\end{array}$ & 34,1 & 0,94 & 0,42 & $\begin{array}{ll}38,7 & 40,9 \\
42,6 & 39,8 \\
40,0 & \end{array}$ & 40,4 & 1,30 & 0,58 \\
\hline $\begin{array}{l}\text { Abies pectinata } \\
\text { Sapin } \\
\text { Weisstanne }\end{array}$ & \begin{tabular}{|l|}
24,6 \\
26,1 \\
25,4
\end{tabular} & 25,5 & 0,58 & 0,29 & $\begin{array}{ll}32,5 \\
33,1 & 32,8 \\
31,2 & \end{array}$ & 32,4 & 0,72 & 0,36 \\
\hline $\begin{array}{l}\text { Solidago virgae aurea } \\
\text { Solidage } \\
\text { Goldrute }\end{array}$ & $\begin{array}{l}37,6 \\
35,7\end{array}$ & 36,6 & 0,95 & 0,67 & $\begin{array}{l}40,5 \\
40,6\end{array}$ & 40,5 & 0,05 & 0,04 \\
\hline $\begin{array}{l}\text { Toutes fleurs } \\
\text { Alle Blüten }\end{array}$ & $\begin{array}{l}33,4 \\
31,8 \\
31,6\end{array}$ & 32,3 & 0,81 & 0,47 & $\begin{array}{l}40,4 \\
42,4 \\
40,6\end{array}$ & 41,1 & 0,90 & 0,52 \\
\hline
\end{tabular}


série composée de dix à douze échantillons peut être effectué en deux heures environ. La précision des résultats obtenus est satisfaisante et en tout cas suffisante pour être exploitée dans le domaine pratique. Notons d'ailleurs que l'analyse enzymatique est très précisément adaptée au dosage sélectif de chacun des deux monosaccharides réducteurs du miel contrairement à l'analyse chimique qui n'a pas toujours un caractère très spécifique, dans le cas, par exemple, du dosage iodométrique des aldoses exprimés en glucose ou du dosage des sucres réducteurs totaux.

Le prix d'un coffret avec l'ensemble de réactifs pour vingt-cinq dosages est de $150 \mathrm{~F}$ T.T.C.*; une analyse répétée, par exemple, deux fois revient donc à $12,50 \mathrm{~F}$ environ en ne tenant compte que du matériel enzymatique employé. En ce qui concerne l'équipement et lorsque l'analyse est effectuée dans le laboratoire d'une petite coopérative apicole, par exemple, alors que les moyens mis en œuvre à ce niveau sont le plus souvent modestes, il suffit de disposer d'un colorimètre ou d'un spectrocolorimètre équipé d'une source lumineuse et de filtres permettant la lecture dans le proche U.V. Notons à ce sujet et pour justifier l'investissement que le spectrocolorimetre sert à plusieurs analyses importantes du miel et notamment au dosage de l'hydroxy-méthyl furfural ainsi qu'à la recherche et au dosage de l'amylase.

Nous proposons donc l'utilisation de la méthode enzymatique pour la recherche et les dosages courants du glucose et du fructose dans le miel. L'analyse par chromatographie en phase gazeuse reste cependant la référence officielle.

Reçu pour publication en octobre 1978. Eingegangen im Oktober 1978.

\section{ZUSAMMENFASSUNG}

Wir haben eine schon bekannte Methode der enzymatischen Mengenbestimmung von Glukose und Fruktose auf den Honig angewandt. Die untersuchten Proben sind Honige bekannter Pflanzenherkunft.

Die Reaktion wird mit spezifischen Enzymen durchgeführt, die von der Firma Boehringer hergestellt und angeboten werden. Die kolorimetrischen quantitativen Bestimmungen müssen mit Sorgfalt erfolgen; sie werden mit einem Spektrokolorimeter im UV-Bereich von $340 \mathrm{~nm}$ durchgeführt.

Die Resultate sind in Tab. 1 angeführt; ihre Reproduzierbarkeit ist annehmbar. Wir schlagen vor, diese Methode routinemässig sowohl in der Forschung wie bei der Bestimmung von Glukose und Fruktose in Honigen anzuwenden.

\section{BIBLIOGRAPHIE}

Bergmeyer H. V., Bernt E., Schmidt F., Stork H. - Methoden der enzymatischen Analyse, in BergMeyer H. V., 2 Ed., Verlag Chemie, Weinheim, 1970, p. 1163.

\footnotetext{
* Prix 1979.
} 
Boehringer. - Glucose, Fructose. Test U.V. pour le dosage du glucose et du fructose dans les aliments. Fiche réf. 139-106. Boehringer Mannheim. G.M.B.H. Biochemica.

GONNET M. (1973). - Deux méthodes colorimétriques simples permettant d'apprécier la teneur en glucose et en fructose des miels. Apidologie 4 (1), 45-55.

Pourtallier J. (1967). - Détermination quantitative des sucres des miels par chromatographie en phase gazeuse. Bull. A picole X (2), 209-212.

Journal OFFICIEL. - Méthodes officielles d'analyse du miel (arrêté du 15-2-1977) n ${ }^{\circ} 77-79,23-4-1977$, 3497-3500. 